\title{
ADMINISTRAÇÃO DE MEDICAMENTO ENDOVENOSO EM PACIENTES DE HOSPITAL DE DOENÇAS INFECTOCONTAGIOSAS: ATUAÇÃO DA ENFERMAGEM
}

Deyse Maria Alves Rocha ${ }^{1}$ Eva Anny Welly de Sousa Brito ${ }^{1}$ Thais Lima Vieira de Souza ${ }^{1}$ Rafaela de Oliveira Mota Maira de Ciero Miranda ${ }^{1}$ Francisca Elisângela Teixeira Lima ${ }^{1}$

\begin{abstract}
https://orcid.org/0000-0002-9311-5557 https://orcid.org/0000-0003-2532-0126 https://orcid.org/0000-0002-2161-2327 https://orcid.org/0000-0003-1586-6678 https://orcid.org/0000-0003-2419-9299 https://orcid.org/0000-0002-7543-6947
\end{abstract}

Objetivo: Avaliar a prática da administração de medicamento endovenosa em pacientes adultos internados em hospital de doenças infectocontagiosas. Método: Estudo descritivo, observacional, desenvolvido em hospital de referência em doenças infectocontagiosas de Fortaleza-CE. Foram realizadas 111 observações de profissionais de enfermagem procedendo à administração de medicamentos endovenosos em pacientes com idade $\geq 18$ anos. Resultados: As ações mais realizadas foram: lê a prescrição médica (100\%), consegue entender a grafia médica (97,2\%) e confere o medicamento relacionado à prescrição (90,6\%). Entretanto, as menos executadas foram: acalmar o paciente (21,6\%), realizar limpeza da bancada (13,5\%), usar equipamento de proteção individual (18,9\%), desinfetar ampola ou frasco ampola (11,7\%). Conclusão: O estudo mostrou diagnóstico situacional da equipe de enfermagem na atuação do preparo e da administração de medicamentos, caracterizando segurança de pacientes no nível sofrivel, segundo o índice de positividade.

Descritores: Infusões intravenosas; Segurança do Paciente; Enfermagem.

\section{INTRAVENOUS DRUG ADMINISTRATION IN HOSPITAL PATIENTS OF INFECTIOUS-CONTAGIOUS DISEASES: NURSING PERFORMANCE}

Objective: To evaluate the practice of intravenous drug administration in adult patients hospitalized for infectious-contagious diseases. Method: Descriptive, observational study, developed at a referral hospital in infectious diseases of Fortaleza-CE. A total of 111 observations were made by nursing professionals on the administration of intravenous drugs in patients> 18 years of age. Results: The most accomplished actions were: read the medical prescription (100\%), can understand the medical script $(97.2 \%)$ and check the medication related to the prescription $(90,6 \%)$. However, the least performed were: calming the patient (21.6\%), cleaning the workbench (13.5\%), using personal protective equipment (18.9\%), disinfect ampoule or ampoule vial (11.7\%). Conclusion: The study showed a situational diagnosis of the nursing team in the preparation and administration of medications, characterizing patient safety at the level of suffering, according to the positivity index.

Descriptors: Infusions; Intravenous; Patient Safety; Nursing.

\section{ADMINISTRACIÓN DE MEDICAMENTOS ENDOVENOSOS EN PACIENTES DE HOSPITAL DE ENFERMEDADES INFECTOCONTAGIOSAS: ACTUACIÓN DEL ENFERMERÍA}

Objetivo: Evaluar la práctica de la administración de medicamentos endovenosa en pacientes adultos internados en el hospital de enfermedades infectocontagiosas. Metodo: Estudio descriptivo, observacional, desarrollado en un hospital de referencia en enfermedades infectocontagiosas de Fortaleza-CE. Fueron realizadas 111 observaciones de profesionales de enfermería procediendo a la administración de medicamentos endovenosos en pacientes con edad> 18 años. Resultados: Las acciones más realizadas fueron: lee la prescripción médica (100\%), consigue entender la caligrafía médica (97,2\%) y confirma el medicamento relacionado a la prescripción (90,6\%). Sin embargo, las menos ejecutadas fueron: calmar al paciente (21,6\%), realizar limpieza de la mesa de trabajo (13,5\%), usar equipo de protección individual (18,9\%), desinfectar la ampolla o frasco ampolla (11,7\%). Conclusión: El estudio mostró diagnóstico situacional del equipo de enfermería en la actuación de la preparación y de la administración de medicamentos, caracterizando seguridad de pacientes en el nivel sufrible, según el índice de positividad.

Descriptores: Infusiones Intravenosas; Seguridad del Paciente; Enfermería.

${ }^{1}$ Universidade Federal do Ceará

Autor Correspondente: Deyse Maria Alves Rocha - E-mail: deysealves1995@gmail.com 


\section{INTRODUÇÃO}

As doenças infecciosas acompanham o homem desde o início da história até os dias atuais, sendo um dos principais problemas de saúde pública. Podem ser causadas por bactérias, vírus ou fungos. Os óbitos a ela associados diminuiu de $35,0 \%$ para $5,0 \%$ nos dias atuais devido principalmente a diminuição de óbitos por diarreia e doenças imunopreviniveis. ${ }^{(1)}$

Umas das principais intervenções realizadas no cuidado ao paciente é a terapia medicamentosa, mas para isso ela deve ser executada de forma correta, caso o contrário, podem ocasionar graves consequências ao indivíduo. (2)

Para promoção da segurança do paciente, é necessário entender que incidente é um evento vindo de atos intencionais ou não intencionais, que acarretou ou pode acarretar danos ao paciente. Assim, têm-se as definições: near miss, quando o incidente não atingiu o paciente; incidente sem dano, quando atinge o paciente, mas não causa dano; e evento adverso, quando o incidente atinge o paciente e causa dano. (3)

A Organização Mundial da Saúde (OMS), visando promover aumento efetivo na segurança de pacientes, elaborou estratégias para melhoria da qualidade do cuidado. Com isso, foram criada seis protocolos básicos de segurança do paciente que englobam as macroáreas: identificação do paciente, prevenção de úlcera por pressão, segurança na prescrição, uso e administração de medicamentos, cirurgia segura, prática de higiene das mãos em serviços de saúde e prevenção de quedas. ${ }^{(4)}$

No Brasil, de acordo com o Instituto para Práticas Seguras do Medicamento, os erros de medicação são a causa de morte de pelo menos 8.000 pessoas anualmente. Os eventos adversos, ocasionados pela administração de medicamentos, correspondem a 7\% das internações hospitalares, equivalente a 840 mil casos por ano. ${ }^{(5)}$

Diante desta problemática, torna-se necessário conhecer as fragilidades envolvidas no processo de preparo e administração de medicamento. (6) Além disso, torna-se imprescindivel o conhecimento da equipe de enfermagem para garantir melhor qualidade da assistência prestada. ${ }^{(7)}$

O processo de administração de medicamentos endovenosos representa uma das funções assistências da equipe de enfermagem. ${ }^{\left({ }^{8}\right)}$ Sendo assim, é de extrema importância o conhecimento técnico-científico da equipe para a oferta de um serviço de segurança e qualidade para o paciente. ${ }^{(9)}$

Por se tratar de uma prática bastante realizada pela equipe de enfermagem, faz se necessário a realização de estudos acerca desta temática. Além disso, "medicação segura" foi o tema escolhido pela OMS para ser o Desafio Global para Segurança do Paciente de 2017. ${ }^{(10)}$

Tem-se como objetivo do estudo avaliar a prática de enfermagem acerca do processo de preparo e administração de medicamento endovenoso em pacientes adultos internados em um hospital de doenças infectocontagiosas.

\section{MÉTODO}

\section{Tipo de estudo}

Estudo transversal, observacional, de abordagem quantitativa.

\section{Participantes da pesquisa}

Participaram do estudo 11 profissionais, quatro enfermeiros e sete técnicos de enfermagem, que atenderam aos critérios de inclusão: ser profissional de enfermagem; atuar no processo de preparo e/ou administração de medicamento por via EV; e estar em serviço nos dias de realização das observações.

\section{Local do estudo}

Estudo desenvolvido em um hospital público de referência em doenças infectocontagiosas de atenção terciária, localizado em Fortaleza-Ceará-Brasil.

\section{Coleta de dados}

Foram realizadas 111 observações sistemáticas do processo de preparo e administração de medicamento por via endovenosa (EV) em pacientes na referida instituição, que atenderam aos critérios de inclusão: ter idade $\geq 18$ anos, ter prescrição de medicamento por via EV e ter recebido administração de medicamento por via EV por um membro da equipe de enfermagem na referida instituição.

A coleta de dados, realizada de janeiro a maio de 2017 , nos turnos manhã, tarde e noite, em dias aleatórios, foi realizada por meio da observação não participante, direta e sistemática das ações antes, durante e após o preparo e a administração do medicamento, por meio de instrumento do tipo checklist, dividido em cinco etapas, com as ações referentes ao preparo e à administração de medicamento endovenoso: comunicação (5 ações); preparo do ambiente e material (7 ações); preparo do medicamento prescrito (6 ações); punção venosa (6 ações); e técnica de administração de medicamento (1l ações). Para cada ação, existiam três alternativas: sim, não e parcial, assinaladas pelas pesquisadoras no momento da observação do procedimento.

\section{Procedimentos de análise dos dados}

Os dados coletados na pesquisa foram armazenados 
em banco de dados produzidos no Microsoft Excel do Windows 2010, processados e analisados por meio da estatística descritiva e fundamentados de acordo com a literatura pertinente à temática.

Para avaliação do desempenho dos profissionais na execução de cada ação do processo de administração de medicamentos por via endovenosa, determinou-se o Índice de Positividade (IP) para qualidade da assistência: desejável (IP = 100\%); adequado (90 a 99\%); seguro (80 a 89\%); limítrofe (71 a $79 \%)$; e sofrivel (<70\%). (11)

\section{Procedimentos éticos}

O estudo foi aprovado por comitê de ética, conforme parecer $\mathrm{n}$ ㅇ 1.986.269. Os profissionais de enfermagem e os pacientes foram orientados quanto aos objetivos do estudo e concordaram, por meio da assinatura do Termo de Consentimento Livre e Esclarecido, em serem observados durante o processo de preparo e administração de medicamento EV.

\section{RESULTADOS}

Os participantes da amostra que receberam administração de medicamentos pela via endovenosa apresentaram as seguintes características sociodemográficas: 33 $(29,7 \%)$ pessoas do sexo feminino e 78 (70,3\%) do masculino. A faixa etária variou de 18 a 77 anos, com média de 36 anos, predominando entre 19 e 39 anos de idade (74,7\%).

Dentre as 111 observações, 103 pacientes receberam uma medicação endovenosa, seis pacientes receberam dois medicamentos EV e dois pacientes, três medicamentos EV, totalizando 121 medicações prescritas.

Tabela 1 - Distribuição do número de ações realizadas na etapa de comunicação para administração do medicamento. Fortaleza, CE, Brasil, 2017.

\begin{tabular}{|c|c|c|}
\hline Realização da comunicação & $\mathbf{N}$ & $\%$ \\
\hline Lê a prescrição médica & 111 & 100 \\
\hline Consegue entender a grafia da prescrição médica & 108 & 97,2 \\
\hline $\begin{array}{l}\text { Confere nome do paciente relacionando com a pres- } \\
\text { crição }\end{array}$ & 53 & 47,7 \\
\hline Explica o procedimento para o paciente & 27 & 24,3 \\
\hline Acalma o paciente & 24 & 21,6 \\
\hline
\end{tabular}

No tocante à leitura e compreensão da prescrição médica, a pesquisa mostrou grau desejável e adequado, sendo $100 \%$ e $97,2 \%$, respectivamente. Este achado pode ser justificado pelo fato de as prescrições estarem na forma digitada e não manual.

Quase a totalidade dos profissionais realizavam as ações de comunicação entre os profissionais da saúde, uma vez que houve a compreensão da prescrição do medicamento, considerando as ações relacionadas à leitura e ao entendimento da prescrição médica.

As ações de comunicação entre profissional e paciente foram classificadas, segundo o Índice de Positividade, como sofrivel, tendo em vista que maioria dos profissionais não realizava as ações expostas.

Tabela 2 - Distribuição do número de ações realizadas no preparo do ambiente e seleção do material para administração do medicamento. Fortaleza, CE, Brasil, 2017.

\begin{tabular}{|c|c|c|}
\hline Preparo do ambiente e material & $\mathbf{N}$ & $\%$ \\
\hline Realiza limpeza da bancada & 15 & 13,5 \\
\hline Dispõe de bandeja ou cuba rim & 98 & 88,2 \\
\hline Usa equipamento de proteção individual & 21 & 18,9 \\
\hline Faz desinfecção da ampola ou frasco-ampola & 13 & 11,7 \\
\hline Separa bolas de algodão com e sem álcool & 60 & 54,1 \\
\hline $\begin{array}{l}\text { Escolhe seringa compativel com o volume a ser } \\
\text { administrado }\end{array}$ & 77 & 69,3 \\
\hline $\begin{array}{l}\text { Seleciona adequadamente a agulha para } \\
\text { aspiração }\end{array}$ & 44 & 39,6 \\
\hline
\end{tabular}

Na etapa de organização do ambiente e seleção do material adequado, têm-se sete ações. Uma ação alcançou desempenho seguro. A limpeza da bancada foi pouco observada, pois a maioria dos profissionais realizava a limpeza no início do plantão ou conforme a necessidade de organização e limpeza da bancada. Em 88,2\% das observações, identificou-se que o profissional dispunha de bandeja ou cuba rim para levar a medicação até o local onde a criança se encontrava, e nas demais vezes o profissional levava os medicamentos em suas mãos. 
Constatou-se que a ação menos realizada foi a desinfecção da ampola ou frasco-ampola, sendo essencial para evitar contaminação do conteúdo da ampola.

Tabela 3 - Distribuição do número de ações realizadas no preparo do medicamento. Fortaleza, CE, Brasil, 2017.

\begin{tabular}{|c|c|c|}
\hline Preparo do medicamento & $\mathbf{N}$ & $\%$ \\
\hline Confere rótulo da medicação com a prescrição & 96 & 86,5 \\
\hline Verifica a integridade dos invólucros & 17 & 15,3 \\
\hline Inspeciona o frasco para observar possíveis & & \\
\hline partículas, alteração da cor, rachaduras ou & 18 & 16,2 \\
\hline Observa a data de validade do medicamento & 9 & 8,1 \\
\hline $\begin{array}{l}\text { Dilui medicação em diluente adequado ao } \\
\text { medicamento }\end{array}$ & 90 & 81,0 \\
\hline Identifica medicação/soro após preparado & 11 & 9,9 \\
\hline
\end{tabular}

A ação "confere o rótulo da medicação com a prescrição" foi executada em $86,5 \%$ das observações, sendo a única ação relacionada ao preparo da medicação classificada como segura. As outras ações relacionadas ao preparo da medicação atingiram índices sofriveis.

A integridade do invólucro e do respectivo conteúdo deve ser observada sempre antes do preparo do medicamento, visto que alterações das características originais do fabricante pode indicar contaminação do medicamento, sendo inadequado para ser administrado, devido ao risco à saúde do paciente.

A validade do medicamento foi pouco observada, tendo em vista que esta ação é realizada pelos profissionais da farmácia, no processo de dispensação do medicamento. Entretanto, para segurança do paciente, recomenda-se conferir a validade antes de usar o medicamento. ${ }^{(12)}$

Tabela 4 - Distribuição do número de ações realizadas na etapa de punção venosa. Fortaleza, CE, Brasil, 2017.

\begin{tabular}{|c|c|c|}
\hline Punção venosa & $\mathbf{N}$ & $\%$ \\
\hline $\begin{array}{l}\text { Seleciona o local adequando relacionado com a } \\
\text { prescrição }\end{array}$ & 101 & 91,8 \\
\hline
\end{tabular}

Aplica o garrote 15 a $20 \mathrm{~cm}$ acima do local da punção venosa

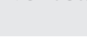

Realiza antissepsia da pele no local a ser puncionado com algodão embebido em álcool a $70 \%$

Introduz o cateter obedecendo ângulo de inserção

Metade dos profissionais realizava a antissepsia do local, sendo classificado como sofrível, segundo o Índice de Positividade. Contudo, recomenda-se a realização da antissepsia da pele sempre antes de realizar a punção venosa. ${ }^{(12)}$

A rede venosa de maior escolha foi a presente na fossa anticubital (67,5\%), seguido pelo arco dorsal da mão (22,5\%), epicranianas (6,3\%) e jugular (1,8\%). Não houve punção nos membros inferiores e nas veias axilares. Os dois pacientes em quem foi realizada a punção em veia jugular faziam tratamento medicamentoso com frequência e se encontravam debilitados, com histórico de rede venosa de difícil punção.

Tabela 5 - Distribuição do número de ações realizadas na técnica de administração de medicamento por via endovenosa. Fortaleza, CE, Brasil, 2017.

\begin{tabular}{|c|c|c|}
\hline Técnica de administração de medicamento EV & $\mathbf{N}$ & $\%$ \\
\hline Retira adornos & 13 & 11,7 \\
\hline Higieniza as mãos & 38 & 34,3 \\
\hline $\begin{array}{l}\text { Confere o medicamento relacionando com a } \\
\text { prescrição }\end{array}$ & 101 & 90,6 \\
\hline $\begin{array}{l}\text { Confere o paciente com o nome que constava na } \\
\text { prescrição }\end{array}$ & 90 & 82,8 \\
\hline $\begin{array}{l}\text { Instala medicação/soroterapia conforme } \\
\text { prescrição médica }\end{array}$ & 111 & 100 \\
\hline Monitora paciente & 31 & 28 \\
\hline $\begin{array}{l}\text { Descarta adequadamente os materiais utilizados } \\
\text { durante o procedimento }\end{array}$ & 109 & 98,1 \\
\hline $\begin{array}{l}\text { Registra/checa imediatamente no prontuário/ } \\
\text { prescrição após administração de medicamento }\end{array}$ & 92 & 82,8 \\
\hline $\begin{array}{l}\text { Registra queixa e/ou reações adversas } \\
\text { apresentadas pelo paciente }\end{array}$ & 20 & 18 \\
\hline Orienta paciente e/ou responsável & 24 & 21,6 \\
\hline Mantém paciente confortável & 104 & 93,6 \\
\hline
\end{tabular}


Precedentemente à etapa de higienização das mãos, faz-se necessária a retirada de adornos (joias, pulseiras e relógio) antes de sua execução, sendo que esta ação foi realizada em apenas 13 das observações (11,7\%), enquadrando-se em desempenho sofrivel.

A higienização das mãos foi realizada pela minoria (34,3\%) dos profissionais, sendo agravado pelo fato desta ação ter sido realizada em menos de 30 segundos. Além disso, não retirar os adornos durante a lavagem, indo de encontro às recomendações da literatura, que indica a duração da higienização das mãos de 40 a 60 segundos, pois o tempo gasto nessa técnica influencia diretamente na redução das bactérias. ${ }^{(13)}$

\section{DISCUSSÃO}

Foi encontrada, neste estudo, predomínio de um medicamento EV por prescrição, fator considerado benéfico na segurança do paciente. Além disso em 100\% das observações houve a leitura da prescrição mé ᄀdica, sendo a realização dessa ação fundamental para impedir a existência de eventos adversos relacionados a medicamentos. Vale ressaltar que erᄀros durante o preparo do medicamento pode gerar consequências para o paciente, como prolongar o tempo de internação, gerar incapacidades, e até levar a morte. ${ }^{(14)}$

Acerca da prática de conferir a prescrição com o nome do paciente, 47,7\% executavam a ação, porém, muitas vezes, perguntavam apenas o primeiro nome, fator de erros de medicação. A confirmação do nome é fundamental para evitar erros, pois existem pacientes com nomes iguais ou parecidos. Além disso essa ação engloba os "nove certos" recomendado pelo Ministério da Saúde, para garantir a administração correta de medicamentos, são eles: paciente certo, medicamento certo, via certa, hora certa, dose certa, registro certo, ação certa, forma certa e resposta certa. ${ }^{(15)}$

A redação manual da prescrição medicamentosa acarreta, muitas vezes, dificuldades de leitura e compreensão, devido à letra ilegivel dos profissionais médicos. Apesar de ser situação frequente no cotidiano da equipe de enfermagem, não é obrigação desta a tradução das prescrições. De acordo com a Resolução Cofen 564/2017, artigo 46, o profissional de enfermagem deverá recusar-se a executar prescrição em caso de erro e/ou ilegibilidade da mesma. (16) $\mathrm{Na}$ etapa de higienização das mãos, das 111 ações observadas, nenhuma obteve resultado satisfatório. De acordo com revisão integrativa que abordava os principais temas relacionados à segurança do paciente, a lavagem de mãos foi discutida em $14,81 \%$ dos artigos encontrados. A prática está diretamente associada à diminuição de infecções hos- pitalares, porém é, muitas vezes, negligenciada. ${ }^{(17)}$

A higienização das mãos, tem como objetivo a redução de patógenos potenciais e risco de infecção hospitalar, logo ela é considerada uma medida básica para o cuidado ao paciente. ${ }^{(13)}$

Em 88,3\% das observações de administração de medicamento, os adornos não eram removidos, em desacordo com a norma regulamentadora № 32, do Ministério do Trabalho, que proíbe o uso de adornos por profissionais da saúde em serviço principalmente aqueles que mantêm contato com agentes biológicos. ${ }^{(18)}$

Os profissionais não executaram uma das ações consideradas mais importantes para o controle de infecção em serviços de saúde, a higienização das mãos. Assim, corrobora-se com estudo que analisa o preparo e administração de medicamentos em idosos hospitalizados, o qual durante a etapa de lavagem de mãos apenas $16,7 \%$ dos profissionais realizaram a etapa. ${ }^{(19)}$ A lavagem das mãos deve ser realizada onde e quando os cuidados são prestados, obedecendo os cinco momentos para a lavagem. ${ }^{(20)}$

O tempo médio necessário para lavagem das mãos é de 40 a 60 segundos. Porém, os resultados deste estudo demostraram que em 100\% das observações, os profissionais realizaram a lavagem das mãos em tempo inferior ao preconizado pela literatura. ${ }^{(13)}$

É importante destacar a necessidade de preparar os materiais e as soluções utilizando técnicas assépticas. Diante do exposto, das sete ações observadas no preparo do ambiente e material utilizado na administração de medicamentos endovenosos, poucos atingiram resultado desejável, caracterizando, assim, um problema identificado, visto que a desinfecção do material e da bancada, a organização e seleção do material adequado para o procedimento e disposição organizada de todo o material são imprescindíveis para o desempenho do procedimento com êxito. ${ }^{(9)}$

No preparo da medicação, foi encontrado resultado seguro em apenas duas ações. A preparação medicamentosa para cada paciente deve ocorrer separadamente, garantindo a conferencia de todos os elementos de identificação da instituição, do paciente, do prescritor e a data estão disponiveis. ${ }^{(15)}$

As falhas relacionadas às normas de biossegurança foram as que mais se destacaram. Um dos cuidados para redução dessas falhas seria a assepsia dos materiais para realização dos procedimentos, como também a lavagem das mãos, a utilização adequada de materiais estéreis e dos equipamentos de proteção individual, medidas simples e de baixo custo, as quais promovem redução de erros de medicação. Contudo, a não adoção destas pode acarretar contaminação ao paciente, gerando infecções e surgimento de 
patógenos resistentes aos fármacos. ${ }^{(16)}$

\section{Limitações do estudo}

Entre as principais limitações, destacou-se a recusa de alguns profissionais em participar do estudo e local de preparação dos medicamentos pequeno, tornando a visualização difícil.

\section{Contribuições para a prática}

O estudo trás importantes contribuições, por analisar a prática de administração de medicamentos e identificar possiveis falhas cometidas pela equipe de enfermagem e assim conscientizar as unidades sobre a importância de uma educação contínua. Uma educação continuada nos hospitais mantém os profissionais atualizados e diminui as chances de erros cometidos.

\section{CONCLUSÃO}

Este estudo permitiu avaliar as ações realizadas pelos profissionais de enfermagem durante o processo de administração de medicamentos endovenosos, de acordo com achados de desempenho desejável, adequado ou seguro: lê a prescrição médica; dispõe de bandeja ou cuba rim, confere rótulo da medicação com a prescrição; dilui medicação em diluente adequado ao medicamento; seleciona o local adequando relacionado com a prescrição; aguarda a evaporação do antisséptico; fixa o cateter adequadamente; confere o medicamento relacionando com a prescrição; confere o paciente com o nome que constava na prescrição; instala medicação/soroterapia conforme prescrição médica; descarta adequadamente os materiais utilizados durante o procedimento; registra/checa imediatamente no prontuário/prescrição após administração de medicamento; e mantem paciente confortável.

As ações com indice de positividade sofrivel foram: explica o procedimento para o paciente; acalma o paciente; realiza limpeza da bancada; usa equipamento de proteção individual; faz desinfecção da ampola ou frasco-ampola; separa bolas de algodão com e sem álcool; escolhe serin- ga compatível com o volume a ser administrado; seleciona adequadamente a agulha para aspiração; verifica a integridade dos invólucros; inspeciona o frasco para observar possiveis partículas, alteração da cor, rachaduras ou vazamentos; observa a data de validade do medicamento; identifica medicação/soro após preparado; aplica o garrote 15 a 20 cm acima do local da punção venosa; realiza antissepsia da pele no local a ser puncionado com algodão embebido em álcool a 70\%; retira adornos; higieniza as mãos; monitora paciente; registra queixa e/ou reações adversas apresentadas pelo paciente; e orienta paciente e/ou responsável.

Dessa forma, possibilitou-se realizar diagnóstico situacional da equipe de enfermagem na atuação do preparo e da administração de medicamentos, que permitirá aperfeiçoamento da qualidade da assistência à saúde, contribuindo para resolução dos problemas identificados, por meio da efetivação de práticas eficazes.

No entanto, destaca-se a necessidade da realização de novos estudos, em outros contextos, com metodologias de avaliação diferenciadas, com a finalidade de tornar o processo mais seguro. Além disso, nota-se a importância da educação permanente para realização de treinamento e sensibilização dos profissionais, para que seja realizada prática segura no processo de administração de medicamento EV.

\section{AGRADECIMENTOS :}

Esta pesquisa contou com a bolsa de estudo do Consetho Nacional de Desenvolvimento Científico e Tecnológico (CNPq) para todas as autoras

\section{CONTRIBUIÇÃO DOS AUTORES:}

Concepção, desenho, análise, interpretação dos dados, redação do artigo, revisão crítica e revisão final: Deyse Maria Alves Rocha, Eva Anny Welly de Sousa Brito, Thais Lima Vieira de Souza, Rafaela de Oliveira Mota, Maira de Ciero Miranda, Francisca Elisângela, Teixeira Lima

\section{REFERÊNCIAS}

1.Woldman EA, Sato AP. Trajetória das doenças infecciosas no Brasil nos últimos 50 anos: um contínuo desafio. Revista de saúde pública. [Internet]. 2016:50:68. [cited 2019 set 27]. Available from:http://www.scielo.br/pdf/rsp/v50/pt_0034-8910-rsp-S1518-87872016050000232.pdf

2.Souza AFR, Queiroz JC, Vieira AN, Solon LGS, Bezerra ELSF. Os erros de medicação e os fatores de risco associados a sua prescrição. Enferm. Foco 2019; 10 (4): 12-16 [cited 2020 fev 27]. Available from: http://revista.cofen.gov.br/index.php/enfermagem/ article/view/1900/598 
3.Lanzillotti LS, Andrade CLT, Mendes W, Seta MH. Eventos adversos e incidentes sem dano em recém-nascidos notificados no Brasil, nos anos 2007 a 2013. Cad. Saúde Pública 2016 Set;32(9).[cited 2019 out 20]. Available from: http://www.scielo.br/pdf/csp/ v32n9/1678-4464-csp-32-09-e00100415.pdf

4.Ministério da Saúde (BR). Protocolos básicos de segurança do paciente. 2016 [cited 2020 fev 27] Available from: https://www. saude.gov.br/acoes-e-programas/programa-nacional-de-seguranca-do-paciente-pnsp/protocolos-basicos-de-seguranca-do-paciente

5.Nascimento MA, Freitas K, Oliveira CGS. Erros na administração de medicamentos na prática assistencial da equipe de enfermagem: Uma revisão sistemática. Ciências Biológicas e de Saúde Unit. Aracaju. Outubro 2016. [cited 2019 out 20]. Available from: https://periodicos.set.edu.br/index.php/cadernobiologicas/article/view/3533/2000

6.Llapa-Rodriguez EO, Silva LSL, Mendes MO, Oliveira JKA, Currie LM. Assistência segura ao paciente no preparo e administração de medicamentos. Rev Gaúcha Enferm. 2017;38(4):e2017-0029. [vited 2019 out 22]. Available from: http://www.scielo.br/pdf/ rgenf/v38n4/1983-1447-rgenf-38-04-e2017-0029.pdf

7.Silva MVRS, Carvalho Filha FSS, Lando GA, Branco TB, Viana LMM, Silva HMC. Cuidados na administração de medicamentos: as responsabilidades dos profissionais de enfermagem. Rev enferm UFPE on line. Recife, 11(Supl. 2):950-8, fev 2017. [cited 2019 out 22]. Available from: https://periodicos.ufpe.br/revistas/revistaenfermagem/article/download/13464/16159

8.Etelvino MAL, Santos ND, Aguiar BGC, Assis TG. Segurança do paciente, uma análise do aprazamento de medicamentos. Enferm. Foco 2019; 10 (4): 87-92.[cited 2020 fev 27]. Available from: http://revista.cofen.gov.br/index.php/enfermagem/article/ view/2251/622

9.Silva MFB, Santana JF. Erros na administração de medicamentos pelos profissionais de enfermagem. Arq. Catarin Med. 2018 out.-dez; 47(4):146-154. [ cited 2019 out 22]. Available from:: http://www.acm.org.br/acm/seer/index.php/arquivos/article/ view/359/321

10.Instituto Brasileiro para Segurança do Paciente. Cultura de Segurança \& Gestão. Medicação Segura é tema escolhido pela OMS para próximo Desafio Global de Segurança do Paciente, 2017. [cited 2019 out 22]. Available from:: https://www.segurancadopaciente.com.br/seguranca-e-gestao/medicacao-segura-e-tema-escolhido-pela-oms-para-proximo-desafio-global-de-seguranca-do-paciente/

11. Murassaki ACY, Versa GLGS, Ballucci Júnior JÁ, Meireles VC, Vituri DW, Matsuda LM. Avaliação de cuidados na terapia intravenosa: desafio para a qualidade na enfermagem. Esc Ana Nery (impr.) 2013 jan-mar; 17(1):11-16. [cited 2019 out 23]. Available from:: http://www.scielo.br/scielo.php?script=sci_arttext\&pid=S1414-81452013000100002

12.Agência Nacional de Vigilância Sanitária (ANVISA). Protocolo de segurança na prescrição, uso e administração de medicamentos, 2013. [cited 2020 fev 27]. Available from:: https://www20.anvisa.gov.br/segurancadopaciente/index.php/publicacoes/item/ seguranca-na-prescricao-uso-e-administracao-de-medicamentos

13.Agência Nacional de Vigilância Sanitária (ANVISA). Segurança do paciente, higienização das mãos; 2013. [cited 2020 fev 27]. Available from:: https://www20.anvisa.gov.br/segurancadopaciente/index.php/publicacoes/item/seguranca-do-paciente-higienizacao-das-maos

14.Mendes JR, Lopes MCBT, Campanharo CRV, Okuno MFP, Batista REA. Tipos e frequência de erros no preparo e na administração de medicamentos endovenosos. einstein (São Paulo). 2018;16(3):1-6.[cited 2020 fev 27] Available from:: http://www.scielo.br/ pdf/eins/v16n3/pt_2317-6385-eins-16-03-eAO4146.pdf

15. Ministério da Saúde (BR). Fundação Oswaldo Cruz. Agência Nacional de Vigilância Sanitária. Protocolo de Segurança na Prescrição, Uso e Administração de Medicamentos. Brasilia: Ministério da Saúde, 2013b. 46p. [cited 2020 fev 26]. Available from: http:// www.hospitalsantalucinda.com.br/downloads/prot_meficamentos.pdf

16.Conselho Federal de Enfermagem. Resolução n 564, de 6 de Novembro de 2017. Novo código de ética de Enfermagem. [cited 2020 fev 26]. Available from: http://www.cofen.gov.br/resolucao-cofen-no-5642017_59145.html

17.Silva ACA, Silva JF, Santos LRO, Avelino FVSD, Santos AMR, Pereira AFM. A segurança do paciente em âmbito hospitalar: Revisão integrativa da literatura Cogitare Enferm. 2016 v. 21 n. esp: 01-09. [cited 2019 out 23]. Available from: https://revistas.ufpr. br/cogitare/article/view/37763

18. Ministério do Trabalho e Emprego (BR). Portaria $n^{\circ}$ 485, de 11 de novembro de 2005. Aprova a Norma Regulamentadora $n=32$ (Segurança e Saúde no Trabalho em estabelecimentos de Saúde); 2005. [cited 2019 out 23]. Available from: https://www20.anvisa. gov.br/segurancadopaciente/index.php/legislacao/item/portaria-n-485-de-ll-de-novembro-de-2005

19.Fernandes BKC, Freitas ABN, Coutinho DTR, Queiroz TA, Freitas MC. Preparo e administração de medicamentos em idosos hospitalizados. 2018. Revista Kairós-Gerontologia, 21(3), 151-163. ISSNe 2176-901X. São Paulo (SP), Brasil. [cited 2020 fev 26]. Available from: http://ken.pucsp.br/kairos/article/view/41453/27913

20.Agência Nacional de Vigilância Sanitária (ANVISA). Manual de referência técnica para higiene das mãos; 2015. [cited 2019 out 23]. Available from: https://www20.anvisa.gov.br/segurancadopaciente/index.php/publicacoes/item/manual-de-referencia-tecnica-para-a-higiene-das-maos7 